\title{
Le buste en marbre de Gustav Mühl
}

\section{Claude Lorentz}

\section{(2) OpenEdition}

Journals

Édition électronique

URL : http://journals.openedition.org/rbnu/952

DOI : 10.4000/rbnu.952

ISSN : 2679-6104

\section{Éditeur}

Bibliothèque nationale et universitaire de Strasbourg

\section{Édition imprimée}

Date de publication : 1 mai 2017

Pagination : 88-93

ISBN : 9782859230661

ISSN : 2109-2761

\section{Référence électronique}

Claude Lorentz, "Le buste en marbre de Gustav Mühl », La Revue de la BNU [En ligne], 15 | 2017, mis en ligne le 01 novembre 2019, consulté le 12 décembre 2020. URL : http://journals.openedition.org/rbnu/ 952 ; DOl : https://doi.org/10.4000/rbnu.952

\section{(C) $10(0$}

La Revue de la BNU est mise à disposition selon les termes de la Licence Creative Commons Attribution - Pas d'Utilisation Commerciale - Partage dans les Mêmes Conditions 4.0 International. 


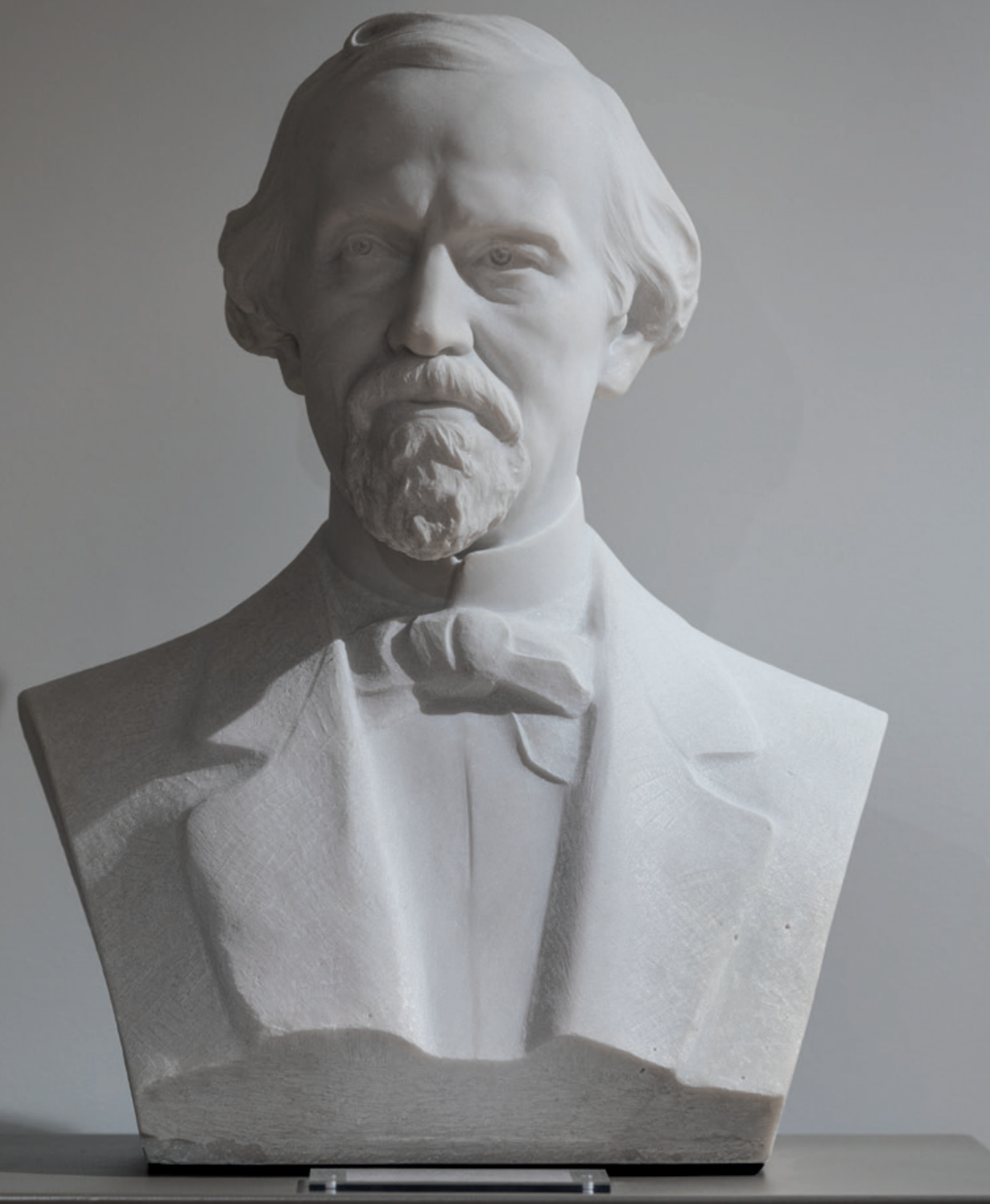




\title{
Le buste en marbre de Gustav Mühl
}

\author{
* \\ PAR CLAUDE LORENTZ
}

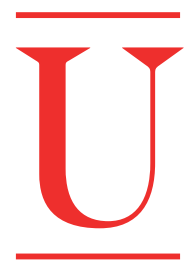

n conservateur de la BNU statufié ! La chose est peu banale. Ce buste en marbre blanc renvoie aux premières décennies de l'histoire de la bibliothèque et à l'une de ses sections emblématiques, celle des alsatiques. Il représente en effet Gustav Mühl (1819-1880), le premier conservateur (« custos ») de cette section entre 1874 et $1880^{1}$.

Rappelons que la Kaiserliche Universitäts- und Landesbibliothek zu Straßburg (KULBS), l'ancêtre de l'actuelle BNU, est officiellement installée le 9 août 1871 tandis que son statut définitif lui conférant la personnalité juridique d'une institution publique intervient avec l'ordonnance impériale du 19 juin $1872^{2}$. La bibliothèque comprend bientôt douze sections selon le système de classification initié par Julius Euting sur le modèle de celui de bibliothèque universitaire de Tübingen où il exerçait précédemment : A : Généralités ; B : Philosophie ; C : Philologie ; D : Histoire ; E : Théologie ; F : Droit ; G : Sciences politiques; $\mathrm{H}$ : Sciences naturelles ; J : Médecine ; $\mathrm{K}$ : Incunables ; L : Manuscrits et $\mathrm{M}$ : Alsatiques. Gustav Mühl devient en 1874 le premier conservateur de cette dernière section ${ }^{3}$.

Né à Strasbourg en 1819, il est le fils cadet d'un officier retraité des armées napoléoniennes et d'une mère issue d'une famille de négociants strasbourgeois. Il effectue sa scolarité au Gymnase protestant où il suit l'enseignement de Jean Geoffroy Schweighaeuser (1776-1844) qui l'éveille à sa vocation littéraire. Après son baccalauréat ès lettres obtenu en 1836, il s'oriente, sous l'influence de son père plutôt que par intérêt personnel, vers des études de médecine. En 1847, il est reçu docteur de la Faculté de médecine de Strasbourg avec une thèse sur le rachitisme mais, attiré par la littérature et bénéficiant de la fortune familiale, il renonce à exercer pour se consacrer aux lettres.

Le jeune Gustav Mühl entreprend alors de nombreux voyages en Allemagne (Stuttgart, Munich, Weimar, Leipzig, Berlin), où il tisse des liens avec des cercles littéraires et savants. Ardent défenseur de la prééminence de la langue et de la culture allemandes en Alsace, il collabore durant les années 1850 aux publications des mulhousiens Frédéric Otte et Auguste Stoeber (Elsässisches Samstagsblatt, Alsatia...) ainsi qu'à plusieurs journaux et revues d'Outre-Rhin dont la Leipziger illustrierte Zeitung. En 1853, il épouse Wilhelmine Candidus, la sœur de son meilleur ami le poète Charles Candidus (1817-1872). Le couple a quatre filles dont deux épousent des universitaires, entre autres l'historien Karl Lamprecht, professeur à l'université de Leipzig. Entré tardivement à la KULBS, en 1874 à l'âge de 55 ans, son temps d'exercice en tant que conservateur des alsatiques est de fait assez court, moins de 6 années, puisqu'il s'éteint le 27 août 1880 . Alors pourquoi un buste en son honneur ?

Dès 1881, un comité constitué à l'initiative du poète Richard Boeckh, réunissant notamment Karl August Barack, le directeur de la KULBS, Adolf Michaelis, le fondateur et directeur de l'Institut d'archéologie classique de la Kaiser-Wilhelms-Universität et les frères Stoeber, œuvre pour l'apposition d'un 
médaillon en bronze à sa stèle funéraire au cimetière Sainte-Hélène de Strasbourg ${ }^{4}$. C'est l'homme de lettres et le partisan de l'assimilation culturelle de l'Alsace au Reich qui est ainsi honoré. Mühl est en effet l'auteur d'une quinzaine de contes, poésies et biographies, dont des portraits du peintre Jean Klein (1803-1855), du sculpteur André Friedrich (1798-1877), de l'archiviste de la ville de Strasbourg Louis Schneegans (1812-1858). Sa bibliographie ${ }^{5}$ mentionne en outre des poèmes d'allégeance dédiés à l'empereur Guillaume $1^{\mathrm{er}}$.

La production du buste intervient vingt ans après ce premier hommage, dans un contexte différent. À l'honneur rendu à l'homme de lettres et ardent défenseur de la culture allemande en Alsace s'ajoute celui rendu au conservateur de la section des alsatiques. Gustav Mühl est en effet, indirectement, à l'origine du premier catalogue imprimé de la section $M$.

Dès la création de la KULBS, la Délégation d'Alsace-Lorraine (Landesausschuss) demande la rédaction d'un catalogue consacré aux collections alsatiques, dont le noyau est constitué par la bibliothèque de l'imprimeur et libraire Friedrich Karl Heitz, riche de 27500 volumes. Le projet traîne durant de longues années faute de financement, de bibliothécaires en nombre suffisant et en raison de désaccords sur la méthode à mettre en œuvre. L'affaire se débloque au tournant du siècle grâce à la création, le 9 novembre 1899, par la veuve de Gustav Mühl, Wilhelmine, d'une fondation alimentée d'un montant de 20000 marks destiné à financer la rédaction et l'impression de ce catalogue. Le gouvernement en accepte le principe et le bibliothécaire Ferdinand Mentz est chargé, en 1902, de sa rédaction sous la direction d'Ernst Marckwald, le successeur de Mühl à la tête de la section $\mathrm{M}^{6}$. En février 1908 , un premier volume est publié sous la direction de Marckwald, Mentz et Ludwig Wilhelm. La page de titre porte la mention «Aus den Mitteln der Mühl'schen Familienstiftung ${ }^{7}$. Le volume comprend en page de garde un portrait de Gustav Mühl précédant quelques pages introductives consacrées à sa vie et à son œuvre. Ce catalogue reprend fidèlement le fichier systématique de la section, qui compte alors 45500 documents dont 2000 volumes de périodiques, des cartes et plans, des documents iconographiques parmi lesquels une importante collection de portraits de personnalités alsaciennes et lorraines. Deux tomes suivront après la guerre, en 1923 et 1926, sous la direction de Wilhelm rejoint par François Ritter. C'est pour marquer cette publication d'importance majeure pour la KULBS qu'à la demande de la famille du défunt conservateur et grâce au reliquat et aux intérêts du capital de la fondation, la bibliothèque décide d'ériger un monument en sa mémoire ${ }^{8}$. Celui-ci prend la forme d'un buste en marbre placé sur un piédestal en syénite.

Une commande est passée au début de l'année 1911 au sculpteur autrichien résidant à Rome Ferdinand Seeboeck (1864-1952). Natif de Vienne, F. Seeboeck a étudié à l'Akademie der bildenden Künste de la capitale autrichienne avant de s'établir à Rome en 1885 . Disposant d'un atelier via Margutta, non loin de la place d'Espagne (piazza de Spagna), centre névralgique du quartier des artistes, il est lié entre autres à la mécène Henriette Hertz (1846-1913), la fondatrice de la célèbre bibliothèque d'art Bibliotheca Hertziana ${ }^{9}$. Spécialisé dans la sculpture de portraits, il est l'auteur de nombreux bustes, tant à Rome qu'en Allemagne où il retourne épisodiquement (il est actif à Baden Baden de 1905 à 1909 notamment).

Le buste de Mühl, d'un poids de $250 \mathrm{~kg}$, est expédié de Rome vers Strasbourg le 28 juin 1911. Il est dévoilé le dimanche 8 octobre de la même année lors d'une cérémonie officielle réunissant, dans le vestibule $\mathrm{du}$ premier étage de la bibliothèque et aux côtés des membres de la famille, la direction et le personnel de la KULBS, des représentants du corps professoral de l'université (les professeurs Laband, Bresslau, Neumann), du monde des arts et des lettres, du gouvernement d'Alsace-Lorraine et de la ville de Strasbourg. À la lecture du compte rendu qu'en dresse le quotidien Strassburger Neueste Nachrichten le lendemain ${ }^{10}$, on perçoit la tonalité éminemment patriotique de la cérémonie. Celle-ci est encadrée par deux séquences musicales exécutées par un quatuor masculin du «Strassburger Männergesangverein » qui entonne Deutsche Heimat en ouverture et In die Ferne du compositeur suisse Friedrich Hegar (1841-1927) en clôture. Dans l'intervalle, se succèdent les discours-hommages du professeur Karl Lamprecht (1856-1915), le gendre de G. Mühl, et de Georg Wolfram (1858-1940), le directeur de la KULBS. Tous deux louent le conservateur poète, chantre du " Deutschtum » et de la petite « Heimat " d'Alsace $^{11}$. Un texte d'hommage est ensuite lu par le 


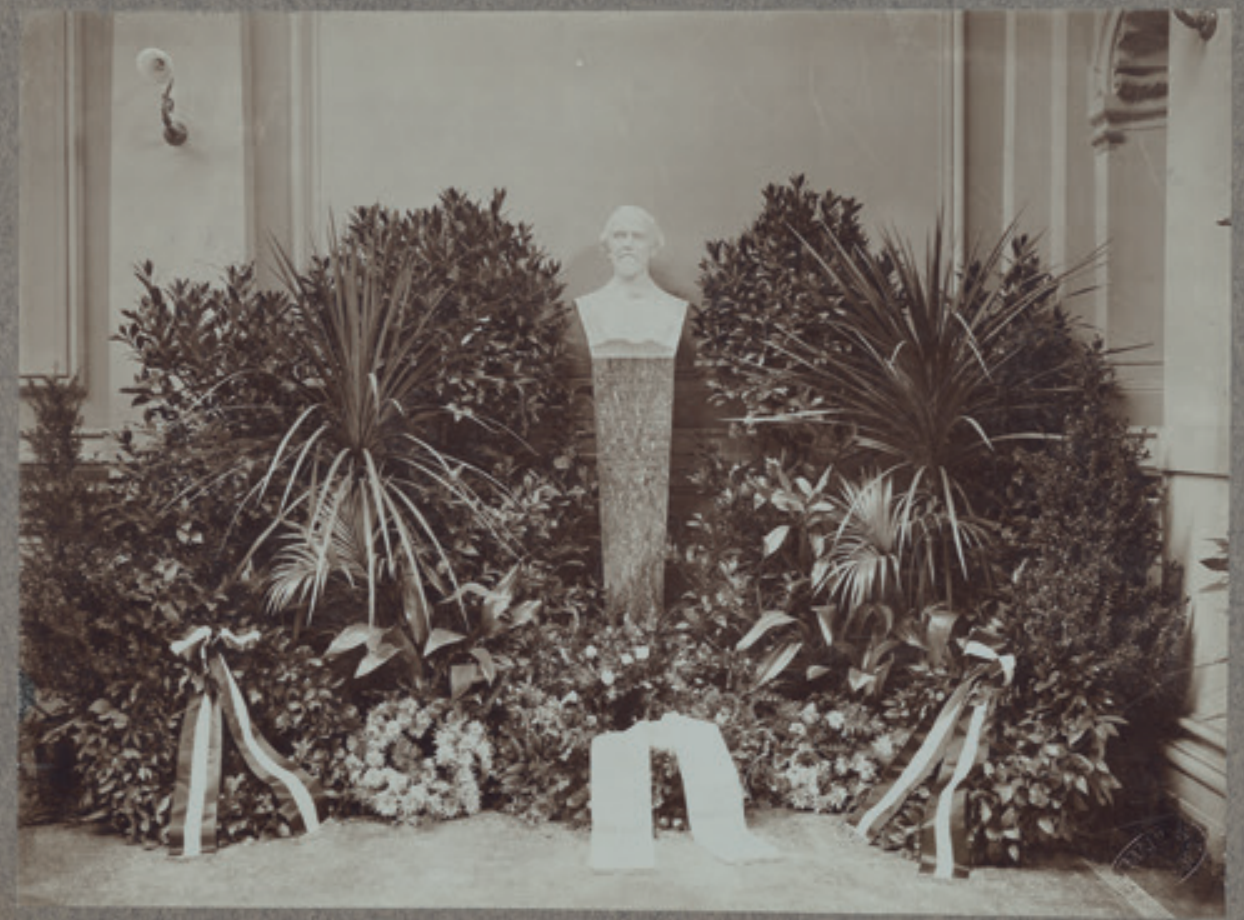

Jules Manias, Buste de Gustav Mühl, photographie, Strasbourg, 1911, 30 × $24 \mathrm{~cm}$.

Le socle en syénite que l'on voit sur cette photographie, et qui a disparu depuis, porte l'épitaphe :

« Gustav Mühl 1819-1880

Wie mit Herz und

Geist dein Streben

Treu für deutche Art gestritten hat gepflegt dein Lied und Leben Rein der Heimat Sinn und Sitten » 


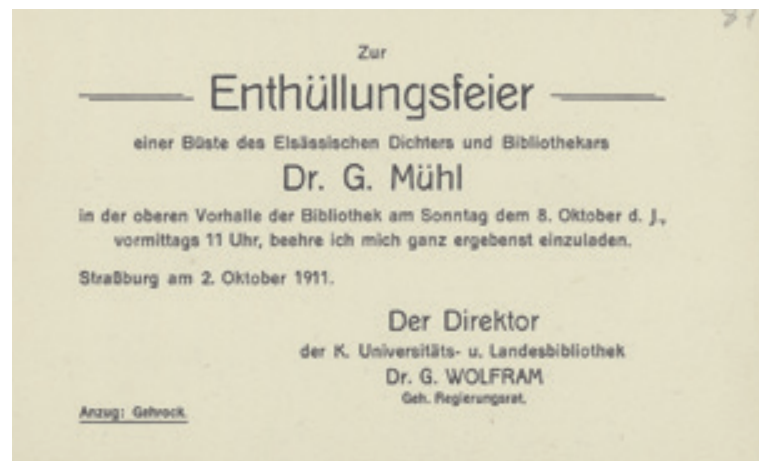

\footnotetext{
Carton d'invitation à la cérémonie de dévoilement du buste à la Kaiserliche

Universitäts- und Landesbibliothek zu Straßburg, le 8 octobre 1911 (archives de la BNU)
}

bibliothécaire de la KULBS, poète et très germanophile Christian Schmitt (1865-1928) ${ }^{12}$, tandis que le photographe Jules Manias (1866-1944), missionné par la direction de la bibliothèque, immortalise l'événement ${ }^{13}$. Le rédacteur des Strassburger Neueste Nachrichten, dans sa recension, accentue encore le caractère patriotique de la cérémonie en établissant un parallèle entre Mühl et le personnage d'Arthur de Gobineau (1816-1882) dont la collection, acquise par la KULBS en 1903, fait l'objet depuis 1906 d'une valorisation dans un petit musée permanent situé au premier étage de la bibliothèque ${ }^{14}$. Il écrit ainsi : " Une exposition Gobineau offre une espèce de pendant à la cérémonie en l'honneur de Gustav Mühl. L'enthousiasme du ci-devant citoyen français Gustav Mühl pour l'art et la poésie allemands trouve un pendant intéressant en la personne de l'écrivain, peintre et sculpteur français Gobineau, qui accorde un rôle de guide à l'Allemagne dans le concert européen des nations [...] Les travaux de toute nature de Gobineau attestent un connaisseur et un homme de grand jugement, et son jugement à propos de la race germanique est précieux pour la valorisation de notre nation et de notre race ${ }^{15}$.

L'auteur fait référence à l'Essai sur l'inégalité des races humaines publié par Gobineau en 1853, essai aux accents pangermanistes proposant une lecture raciale de l'histoire humaine, largement ignoré en France mais dont la réception en Allemagne fut et demeurait alors vive, comme on le constate à la lecture de ces lignes. Le contexte général en cette fin d'année 1911 est, de fait, propice à de tels élans patriotiques, y compris dans un quotidien tel que les Strassburger Neueste Nachrichten pourtant considéré comme modéré. On se situe en effet quelques mois après l'adoption, en mai 1911, de la nouvelle loi constitutionnelle, plus libérale certes que le régime précédent, mais qui ne satisfait en réalité aucune force politique en présence, avec en toile de fond, comme le souligne l'historien François Igersheim, « un contexte européen marqué par l'accentuation de l'antagonisme franco-allemand et les mobilisations nationales [...], la course aux armements et les répercussions des guerres balkaniques ${ }^{16}$. Les affaires de Graffenstaden en 1912, puis de Saverne l'année suivante, constituent bientôt les épisodes paroxystiques de ces tensions nationales en Alsace.

Ainsi, l'histoire de ce buste du poète et conservateur de bibliothèque Gustav Mühl nous entraîne au cœur des enjeux politiques et culturels de l'Alsace du début du $20^{\mathrm{e}}$ siècle et démontre, s'il était nécessaire, que la BNU fut également, au cours de son histoire singulière et au regard de son rôle culturel et scientifique, le théâtre de ces enjeux.

Le buste de Gustav Mühl, sans son socle originel qui a disparu, est visible dans les réserves visitables de la BNU, accessibles au public dans le cadre des visites guidées du samedi matin (voir les modalités sur le site internet de la BNU : http://www. bnu.fr/infos-pratiques/visiter-la-bnu). 


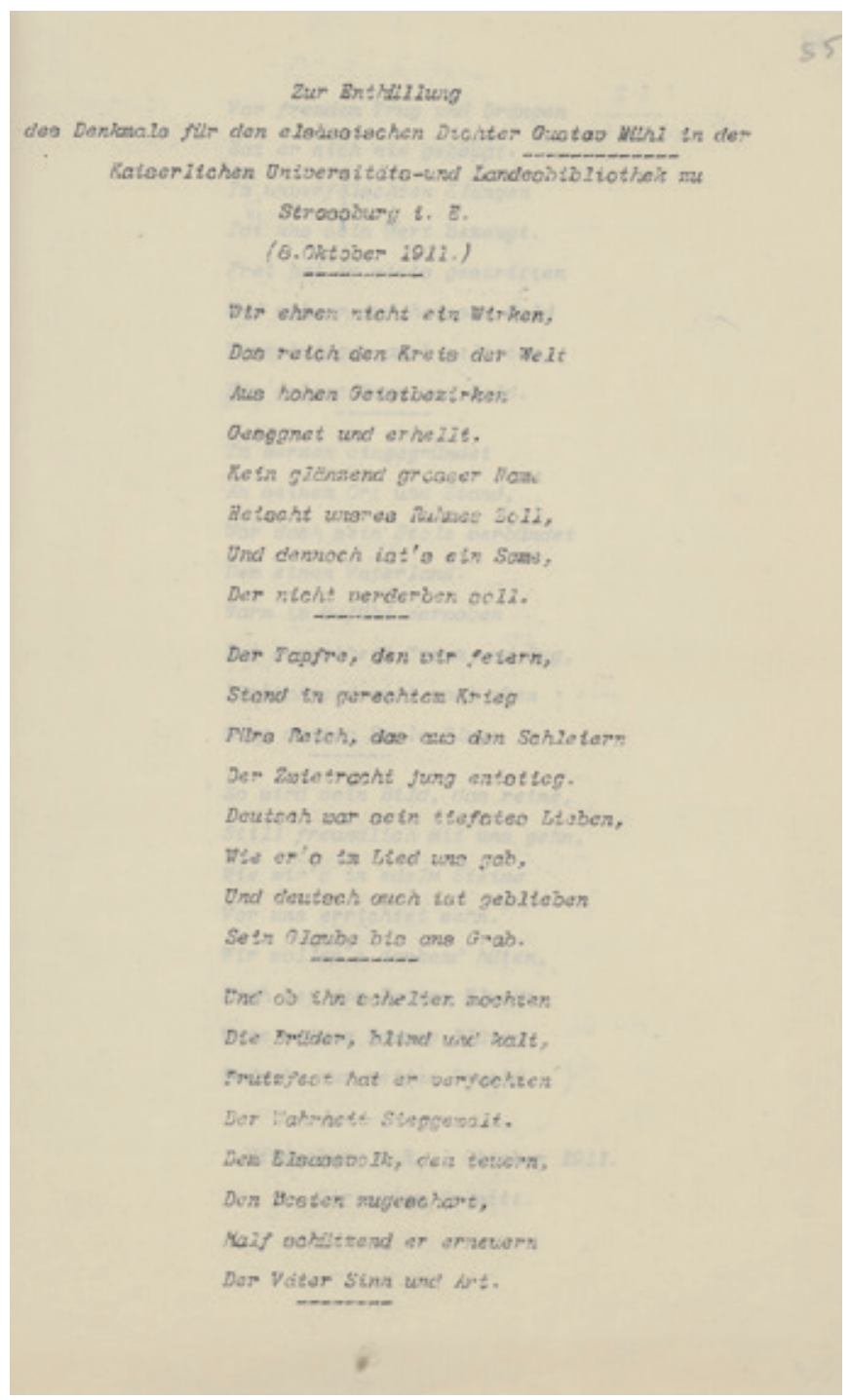

Christian Schmitt, « Zur Enthüllung des Denkmals für den elsässischen Dichter Gustav Mühl in der Kaiserlichen Universitäts- und Landesbibliothek zu Straßburg i. E. (8. Oktober 1911) », Strassburg i. E., 1. Oktober 1911 (tapuscrit ; archives de la BNU)

\section{NOTES}

1- Notices « Mühl Gustave » dans le Nouveau dictionnaire de biographie alsacienne, $\mathrm{n}^{\circ} 27,1996$, p. 2730-2731, et dans Encyclopédie d'Alsace, vol. 9, p. 5321-5322.

2- Frédéric Barbier (sous la dir. de), bibliothèques strasbourg origines $X X I^{e}$ siècle, Paris, Éditions des Cendres, Strasbourg, Bibliothèque nationale et universitaire, 2015, p. 179-256

3- François Igersheim, L'Alsace et ses historiens 1680-1914. La fabrique des monuments, Strasbourg, Presses universitaires de Strasbourg, 2006, p. 258-269

4- Ibid. et archives de la BNU : AL 50, 29 et AL 50, 30

5- La liste des œuvres de Mühl figure dans : Katalog der ElsassLothringischen Abteilung, bearbeitet von Prof. Dr. Ernst Marckwald, Dr. Ferdinand Mentz und Ludwig Wilhelm, I, Strasbourg, Selbstverlag der Bibliothek, 1908-1911, p. XII.

6- François Igersheim, op. cit., p. 267-269

7- «Réalisé grâce aux moyens mis à disposition par la Fondation familiale Mühl».

8- Archives de la BNU : AL 51, 22. L'auteur adresse ses remerciements à Daniel Bornemann pour le déchiffrement des manuscrits des archives de la BNU.

9- Aujourd'hui Max-Planck-Institut für Kunstgeschichte.

10- Strassburger Neueste Nachrichten du 9 octobre 1911

11- Le discours de Georg Wolfram est publié en 1912 : Rede bei der Enthüllunsfeier der Büste des elsässischen Dichters Gustav Mühl am 8. Oktober 1911 gehalten von Geh. Regierungsrat Dr. Georg Wolfram, Direktor der Kaiserl. Universitäts- $u$. Landesbibliothek, Strasbourg et Leipzig, Joseph Singer, 1912 (cote BNU : M.27.031).

12- Reproduit dans la publication citée en note 11 et dans les Strassburger Neueste Nachrichten du 9 octobre 1911. Le texte figure également sous forme de tapuscrit dans les archives de la BNU : AL 51, 22. Sur Christian Schmitt, voir la notice dans le Nouveau dictionnaire de biographie alsacienne, $\mathrm{n}^{\circ} 33,1999$, p. 3481-3482.

13- BNU : P. MUHL. G. 400

14- Voir : Christophe Didier, Isabelle Didier et Pierre-Louis Rey, «Le fonds Gobineau ", in Impressions d'Europe. Trésors de la BNUS entre France et Allemagne, Strasbourg, BNU, 2003, p. 64-69.

15- «Eine Gobineau-Ausstellung liefert eine Art von Gegenstück zu der Gustav Mühl-Feier. Die Begeisterung des einstigen Franzosen Gustav Mühl für deutsche Art und Dichtung findet ein interessantes Gegenstück in dem französischen Schriftsteller, Maler und Bildhauer Gobineau, der Deutschland die führende Rolle im Konzerte Europas einraümt [...] Gobineaus Werke aller Art weisen ihn als einen Kenner und Beurteiler höchster Art, und sein Urteil über die germanische Rasse ist wertvoll für die Wertung unserer Nation und Rasse ».

16- François Igersheim, L'Alsace politique 1870-1914, Strasbourg, Presses universitaires de Strasbourg, 2016, p. 173 